\title{
Control of inflammation by integration of environmental cues by regulatory $T$ cells
}

\author{
Ashutosh Chaudhry and Alexander Y. Rudensky
}

Howard Hughes Medical Institute and Immunology Program, Memorial Sloan-Kettering Cancer Center, New York, New York, USA.

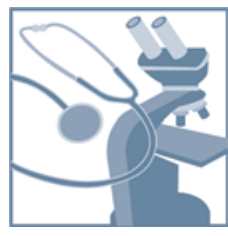

Tregs have been implicated in control of homeostasis in the immune system and beyond. These cells restrain inflammatory responses to self antigens, commensal microorganisms, allergens, and pathogens and adapt their homeostatic and functional capabilities to a particular environment. In this review, we discuss a general model of integration of environmental cues by Tregs in which specialized Treg homeostatic, migratory, and suppression programs are established in dynamically changing inflammatory environments by maintaining an optimal threshold of activation of transcription factors involved in regulation of the corresponding type of effector immune responses.

\section{Inflammation and immune responses}

Immune response-associated inflammation is required for restoration of tissue homeostasis during infection or injury. An inflammatory response is initiated when sensors, predominantly present on phagocytic cells of the innate immune system, detect an altered cellular state or function. Although infectious agents constitute the most commonly described inducers of inflammation, tissue damage during injury, cancer, and a wide variety of autoimmune, metabolic, and degenerative disorders also perturb normal tissue homeostasis and promote inflammatory responses (1). Activation and the subsequent generation of a protective $\mathrm{T}$ cell response is a key component of inflammation-associated immune reaction to pathogens. Distinct types of invading pathogens require different strategies for their elimination or curtailment, a predicament that necessitates generation of distinct kinds of protective inflammatory immune responses. A typical pathogen usually triggers a set of innate immune molecular sensors (e.g., TLRs, Nod-like receptors [NLRs], inflammasomes, retinoic acid-inducible gene I [RIG-I], melanoma differentiation-associated gene 5 [MDA-5], dectin-1), which facilitate elaboration of particular soluble and membranebound mediators and thereby guide differentiation of $\mathrm{T}$ and $\mathrm{B}$ lymphocytes into a distinct effector cell type.

This general principle is based on a large body of experimental data which indicate that naive $\mathrm{CD} 4^{+} \mathrm{T}$ cells differentiate primarily into IFN- $\gamma$ - and TNF- $\alpha$-producing effector Th 1 cells during infection with a virus or intracellular bacteria; IL-4-, IL-5-, and IL-13producing Th2 cells and IL-17- and IL-22-producing Th17 cells are elicited during infection with helminths and with extracellular bacteria, yeast, or fungi, respectively (2). Distinct sets of cytokines and other soluble and membrane-bound factors displayed by these cells in turn promote matched effector function of other cells of the innate and adaptive arms of the immune system. Thus, these $\mathrm{T}$ cell subsets serve not only as effectors, but also as principal amplifiers of an appropriate inflammatory response, which ultimately leads to pathogen clearance or sequestration. We must note that this is a rather simplistic view of immunity to infections, since it is likely that additional types of effector responses have not been recognized yet, and even three main classes of effector

Conflict of interest: Alexander Y. Rudensky is a member of the scientific advisory board of Tempero Pharmaceuticals.

Citation for this article: J Clin Invest. 2013;123(3):939-944. doi:10.1172/JCI57175. responses could be further subdivided. Moreover, pathogens can elicit "mixed" responses, which may reflect the aforementioned unrecognized response types, previous history of encounter with pathogens, and complex three-way interactions with pathogen and commensal microbiota. Finally, "polyfunctional" T cell responses might also be explained by a need for a protective inflammatory response to ensure not only pathogen containment, but also disposal of the damaged tissue and its repair.

Upon pathogen challenge, signals provided by innate immune cells in the form of peptide-MHC complexes and costimulatory molecules as well as cytokines induce expression of lineage-specifying transcription factors in $\mathrm{T}$ cells that facilitate acquisition of a characteristic gene signature supporting distinct effector, homing, and homeostatic modalities. Cytokine sensing constitutes a major skewing determinant in this process; cytokine receptors work by phosphorylating members of the STAT protein family that are latent transcription factors normally resident in the cytoplasm. Activated STAT proteins undergo nuclear translocation and modulate gene expression. Cytokine-mediated STAT activation drives the expression of lineage-defining factors: T-bet during Th1 differentiation, Gata3 during Th2 differentiation, and orphan nuclear receptor ROR $\gamma t$ during Th17 cell differentiation. This mechanism couples microenvironment sensing with effector $\mathrm{T}$ cell fate decision. Distinct combinations of cytokine-STAT-Th signature transcription factors have been implicated in differentiation of each $\mathrm{T}$ cell lineage, and paucity in any of these molecules results in impaired differentiation of the corresponding Th effector type (2).

As we noted above, the "canalization" of the effector immune responses is explained by the ability of Th cell subsets to support feed-forward loops driving a distinct type of protective immune response. For example, obligate intracellular bacteria or viruses trigger innate immune cell production of IFN- $\gamma$ and IL-12, which ultimately leads to activation of T-bet that directs Th1 cell generation. Production of IFN- $\gamma$ by Th 1 cells that augments activation of IFN- $\gamma$ - and IL-12-producing innate immune cells as well as differentiation of $\mathrm{CD}^{+} \mathrm{T}$ cells into effector CTLs (3). Likewise, production of IL-4 by innate cells triggered in response to parasitic worms activates the Stat6-Gata3 axis-dependent differentiation of Th2 cells, which in turn secrete IL-4, IL-5, and IL-13, cytokines that further mobilize innate immune cells, such as eosinophils and basophils and IgE production by B cells required for effective parasite expulsion (4-6). 
Optimal generation of Th17 cells, abundant at mucosal surfaces of the gastrointestinal tract, is dependent on synergistic action of Stat3-activating cytokines IL-6, IL-21, and IL-23 with TGF- $\beta$ to trigger ROR $\gamma$ t expression $(7,8)$. Th17 cell differentiation can be initiated upon recognition of infected apoptotic $\mathrm{T}$ cells and is substantially affected by the composition of the intestinal microbiota whose products act on intestinal dendritic cells $(9,10)$. IL-17, IL-22, and IL-21 secreted by Th17 cells mobilize neutrophils and act on epithelial cells, potentiating synthesis of anti-microbial peptides as well as tissue remodeling and repair $(11,12)$. In addition, Th17 cells can also facilitate B cell responses (13). Furthermore, a Th17-related cell type, so-called Th22 cells producing IL-22, yet lacking IL-17, is found in skin in both mice and humans and is particularly prominent in psoriatic lesions (14-16). IL-22 production is critically dependent on transcriptional activity of another nuclear receptor, aryl hydrocarbon receptor (AHR) (17). Besides these effector $T$ cell types, a distinct subset of IL-21 producing follicular $\mathrm{T}$ helper (Tfh) cells, whose differentiation is dependent upon transcription factor Bcl6, supports germinal center B cell class-switch recombination (18).

In addition to Th1-, Th2-, or Th17-mediated inflammation induced upon exposure to commensal microorganisms, pathogens, or their products, noninfectious tissue injury such as ischemia results in sterile inflammation associated with both myeloid and lymphoid cell recruitment and activation. This pathway serves to promote tissue repair and restoration and to prevent infection of the affected tissue by opportunistic pathogens (1). Alterations in cellular physiology and function due to disturbance in metabolic balance, stress response to a variety of stimuli, autoimmune disorders, and cancerous lesions result in inflammation whose physiological role is to restore tissue homeostasis. However, the molecular sensors and the signaling cascades capable of detecting and responding to sterile inflammation are not well understood.

Although inflammation represents a powerful mechanism for controlling infection and maintaining normal tissue function, it can have severe deleterious effects if left unrestrained. Uncontrolled activation or impaired dampening of inflammatory responses during the resolution phase can result in permanent impairment of tissue function and chronic inflammation; unchecked Th1 and Th17 responses underlie several chronic autoimmune and inflammatory disorders including colitis, multiple sclerosis, psoriasis, rheumatoid arthritis, and inflammatory bowel disease (IBD), and dysregulated Th2 responses are involved in asthma and atopy (2). Multiple regulatory circuits acting as cell-intrinsic negative feedbacks of signaling pathways, soluble mediators operating in a paracrine and autocrine manner, and suppression mediated by Tregs restrain a wide spectrum of inflammatory responses and sustain homeostasis at tissue and organismal levels.

\section{Treg-mediated control of inflammatory responses}

Tregs, specified by the expression of transcription factor Foxp3, are dedicated to the task of restraining activation and responses of both innate and adaptive immune cells. In line with the central role of Tregs in immune homeostasis, deletion or loss-of-function mutations in the Foxp3 gene result in multiorgan autoimmune disorder in both humans and mice (19-21). The spontaneous deregulation of diverse types of inflammatory responses in Foxp3deficient animals suggests that Tregs are required to maintain normal immune homeostasis. This finding raises a question: are the responses to self-antigens, commensal microorganisms, and pathogens controlled by Tregs via a universal mechanism of suppression, or do these cells utilize modular programs of suppression tailored to control distinct kinds of inflammatory responses?

Tregs express a diverse range of cell surface and secreted molecules capable of suppression (e.g., CTLA-4, TGF- $\beta$, IL-10, TIGIT, CD39, CD73, and granzymes). Some studies suggest that Tregs could control effector $\mathrm{T}$ cells by acting on dendritic cells, by inducing direct cytolysis of activated cells, or by serving as a cytokine sink, thereby arresting proliferation of immune effector cells (22). Although these effector mechanisms taken in isolation can afford a certain degree of immunomodulation, recent studies strongly imply that none of these mechanisms can singly account for Tregmediated immune regulation. For example, selective deficiency in IL-10 production in Tregs results in increased inflammatory responses in mucosal surfaces and skin, but not in systemic widespread inflammatory lesions (23). In fact, as we describe below, recent work suggests that Tregs employ distinct suppression mechanisms depending on the context as well as the location of inflammatory response.

The notion that Tregs adapt their suppression program to a particular inflammatory milieu is based on functional studies of transcription factors, prominently the members of interferon regulatory factor (IRF) and STAT families, which are activated by defined environmental cues. IRF4, a transcription factor required for Th2 and Th17 differentiation, is directly regulated by Foxp3 and is thus highly expressed by Tregs. Conditional ablation of IRF4 in Tregs revealed a selective dysregulation of unprovoked autoreactive Th2 responses characterized by increased production of Th2 cytokines, IL-4-dependent Ig isotype class switching, and pronounced plasma cell generation and accumulation in target tissues. Furthermore, IRF4 was found to associate with Foxp3, and this interaction resulted in cooperative regulation of gene expression (24). One of the molecules targeted by this Foxp3-IRF4 complex was inducible $\mathrm{T}$ cell costimulator (ICOS), which has been previously shown to be required for differentiation on Th2 cells (25). Moreover, expression of certain signature Th2 genes, such as c-maf, CCR9 and IL-33 receptor (T1/ST2), was diminished in IRF4-deficient Tregs. These results suggest that IRF4 might be involved in regulation of a transcriptional module shared by both Th2 and Tregs. Recruitment of IRF4 by Foxp3 probably modifies this module to facilitate suppression of Th2 responses (24). In addition, IRF4 and BLIMP-1 were proposed to facilitate generation of Treg effector cells (26). Recent studies have also implicated Gata-3 in regulation of Foxp3 expression, Treg homeostasis at sites of inflammation, and control of Th2 inflammation at mucosal sites (27-29).

Similarly, Treg-specific ablation of Stat3, a key transcription factor for Th17 cell differentiation, led to uncontrolled Th17 cell responses (30). Analogous to IRF4, upon activation, Stat 3 binds to Foxp3, and this interaction modulates gene expression. Tregs lacking Stat 3 show decreased expression of cytokine receptors known to be involved in Th17 cell differentiation such as IL-6R and IL-1R (31). This lower expression of IL-6R and IL-1R suggests that, akin to IL-2, Tregs might be modulating Th17 development by acting as a sink for IL- 6 and IL-1. Furthermore, Stat3-deficient Tregs produce factors such as TGF- $\beta 1$ and IL- 6 that can drive Th 17 differentiation and display lower levels of CCR6, a chemokine receptor required for migration to sites of Th17 inflammation. Taken together, these defects suggest that Stat 3 can control 


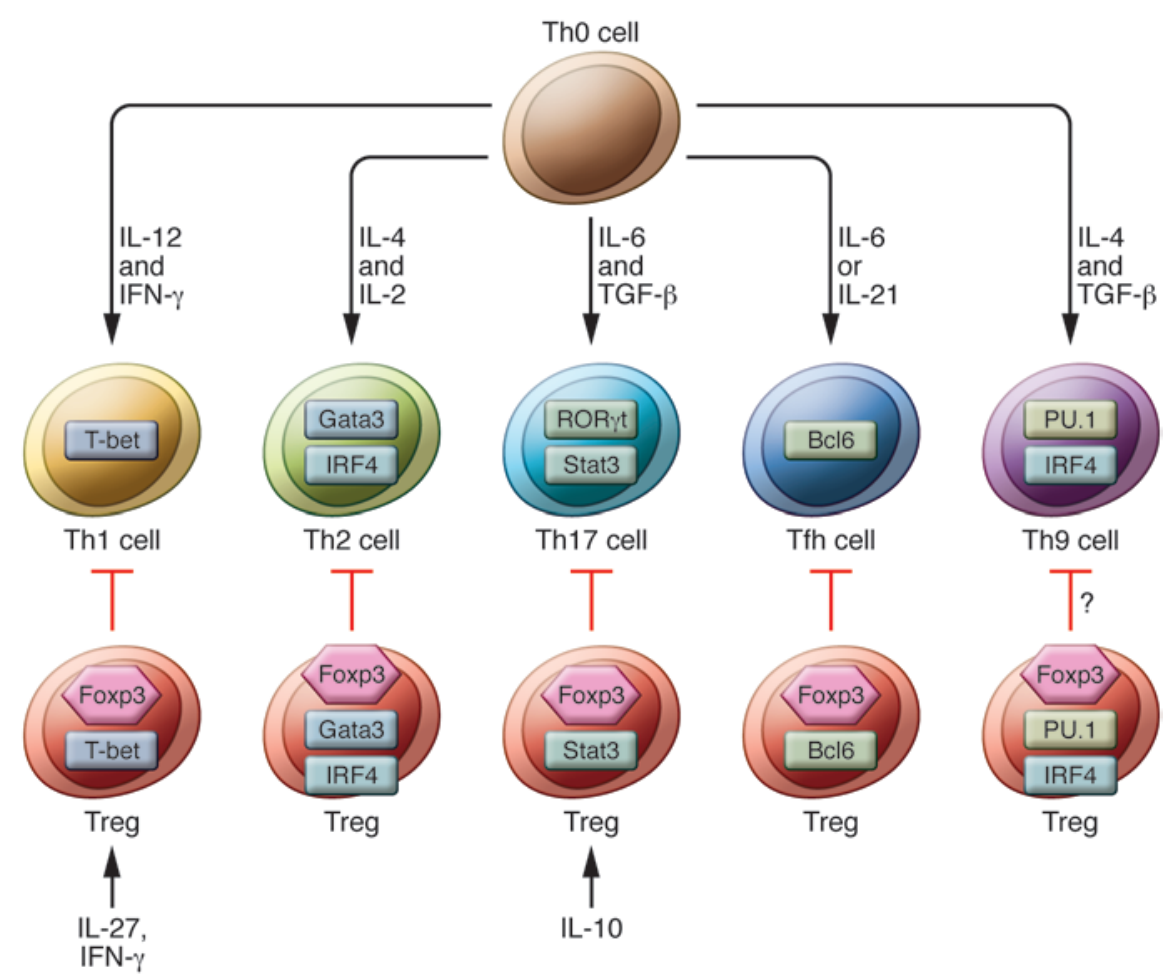

\section{Figure 1}

Peripheral naive CD4+ $\mathrm{T}$ cell differentiation is controlled by cytokine signaling. Th1 cell differentiation is driven by IL-12 and IFN- $\gamma$, Th2 cell development is dependent on IL-4, IL-6 in combination with TGF- $\beta$ mediates Th17 differentiation, and Tfh cell differentiation requires Stat3-inducing factors, such as IL-6 or IL-21, while Th9 cells generation is dependent on IL-4 and TGF- $\beta$. Genetic evidence suggests that coopting of factors required for effector $T$ cell differentiation by Foxp3 endows Tregs to tailor their suppression program for control of the corresponding type of immune responses. Activation of a Th1 suppression module in Tregs is dependent on IFN- $\gamma$ and IL-27, while Th17 suppression requires IL-10-mediated Stat3 activation in Tregs. multiple facets of Treg-mediated suppression of Th17 responses, and therefore further analysis of gene targets that are coregulated by Stat 3 and Foxp 3 would be very informative.

Furthermore, expression of T-bet in Tregs enables them to migrate and proliferate as well as accumulate at the sites of Th1 responses, and Tregs lacking T-bet expression were selectively impaired in regulation of Th1, but not Th2 or Th17, responses (32). However, unlike in IRF4 or Stat3 deficiency, chimeric mice harboring T-bet-deficient Tregs did not show spontaneous immune-mediated inflammation, and the defect is only uncovered upon challenge with agents capable of triggering Th1 responses and under conditions that require Treg expansion.

This symmetrical mechanistic requirement for elaboration of a particular immune effector function and its suppression has been also extended to Treg-mediated control of Tfh cells in three studies, which suggests that increased Bcl6 expression in Tregs allows them to enter germinal centers and to control bystander, presumably self-reactive, antibody responses (33-35). Recent studies showed an additional mode of $\mathrm{T}$ cell activation in response to a combination of IL- 4 and TGF- $\beta$, leading to generation of Th2-related IL-9-producing $\mathrm{CD}^{+} \mathrm{T}$ (Th9) cells, which have been associated with several immune-mediated allergic pathologies. The development of Th9 cells depends on expression of transcription factors IRF4 and PU.1, and it remains to be seen whether Tregs exhibit a similar transcription factor(s) requirement and specialized suppressor mechanisms to control them (refs. 36, 37 and Figure 1).

Together, these results suggest that Tregs exposed to distinct inflammatory environments exhibit heterogeneity resulting from activation of some of the same transcriptional regulators that facilitate responses of immune effector cells. Whether effector $\mathrm{CD}^{+}$ $\mathrm{T}$ cell-specific transcription factors allow Tregs to be equipped with selective migration properties and support their survival in a particular environment or strengthen certain Treg suppression capacities specialized for the corresponding type of immune responses - or both - remains unclear. Nonetheless, these findings strongly support the concept of symmetry in the integration of environmental cues by Tregs and immune effector cells.

\section{The role of cytokines in Treg function}

The signals that trigger lineage-specific suppression programs have not been fully elucidated. Stat 3 is activated downstream of proinflammatory cytokines IL-6, IL-21, and IL-23, which play a crucial role in Th17 cell differentiation and response, as well as IL-10, known to restrain Th17 cell-mediated inflammation. We recently demonstrated that Treg suppression of Th17 responses is dependent on sensing antiinflammatory cues dependent on IL-10 (38). Several different cell types, including T cells upon activation, are known to produce IL-10 (39). These findings imply that in this context, Tregs work as amplifiers of negative regulators elicited by immune effector cells rather than using negative feedback regulation induced by an inflammatory environment. Thus, in analogy with augmentation of effector immune responses by distinct types of Th cells, Tregs act as amplifiers of negative regulatory circuits of immune effector cells to restrain Th17 cell-mediated inflammation.

In effector $\mathrm{T}$ cells, expression of $\mathrm{T}$-bet is induced by proinflammatory cytokines IL-12 and IFN- $\gamma$. It has been reported that loss of IFN- $\gamma$ receptor (IFN- $\gamma$ R) as well as Stat 1 in Tregs leads to a reduction in the number of cells expressing CXCR3 (chemokine [C-X-C motif] receptor 3, a direct T-bet target), suggesting that T-bet in Tregs is triggered upon exposure to IFN- $\gamma(40)$. Genome-wide mapping of permissive and inhibitory histone modifications across various cytokine loci revealed that the IL-12R $\beta$ gene is epigenetically silenced in Tregs and functional IL-12R is not effectively expressed in Tregs in vivo. Nevertheless, in vitro Tregs have been shown to upregulate T-bet in response to IL-12, suggesting that under some conditions IL-12 might act on Tregs (40). 
A

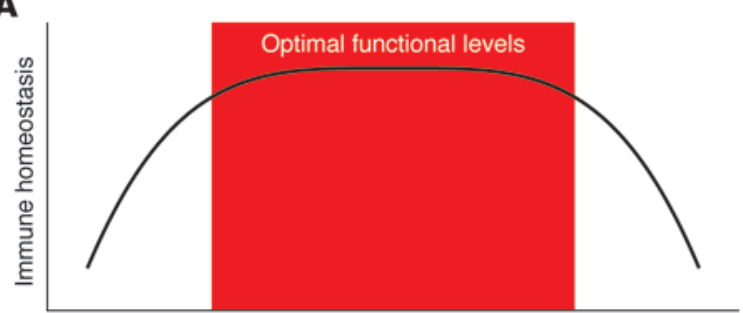

Treg-activated Stat level

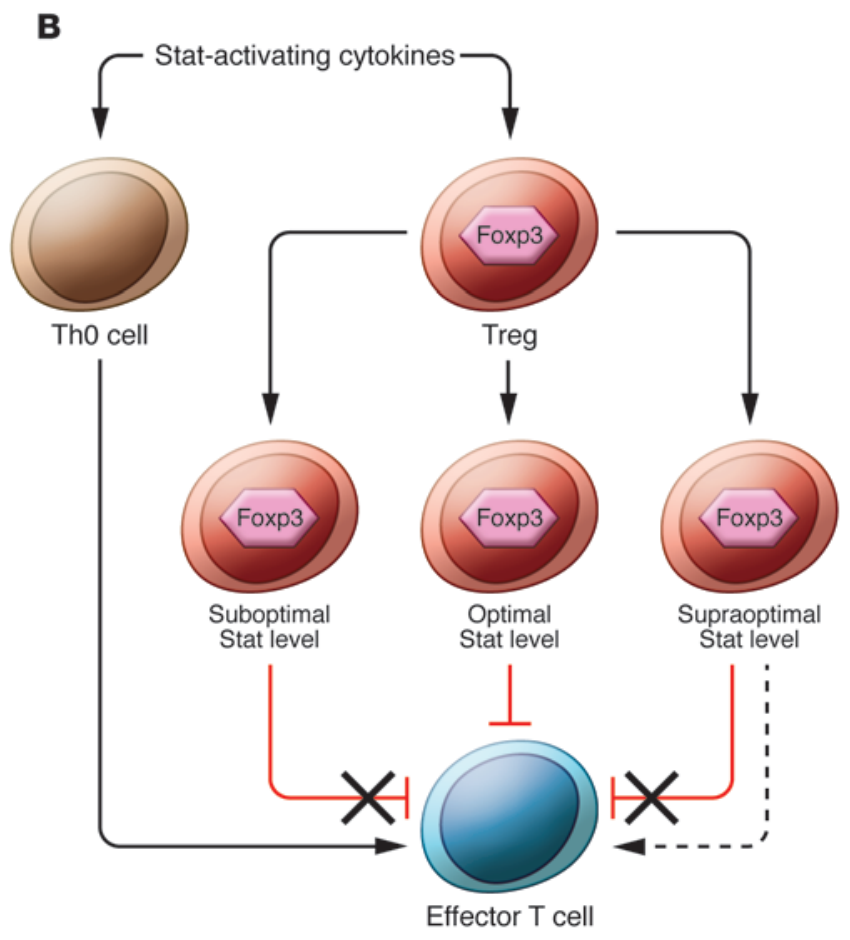

Figure 2

Stat expression in Tregs is required for the ability of Tregs to suppress immune responses. (A) An optimal window of Stat activation in Tregs is required for immune homeostasis. (B) Stat-activating cytokines induce activation of Tregs when Stat levels are optimized. Genetic evidence indicates that sub- or supraoptimal Stat levels result in immune-mediated pathology.

These results suggest that multiple signaling pathways, such as those triggered by IFN- $\gamma \mathrm{R}$, may be responsible for activation of $\mathrm{T}$-bet in Tregs in secondary lymphoid organs. However, at mucosal sites, IL-27, but not IFN- $\gamma$, plays a major role in induction of CXCR3 and T-bet as well as IL-10 in Tregs and allows them to limit inflammatory Th1 responses at local sites (41).

The cytokine signals that enable Tregs to suppress Th2 responses are unknown. The expression of IRF4, requisite for Treg-mediated restraint of Th2-dependent inflammation, is regulated by TCR as well as by Foxp3 $(24,42)$. Although other extracellular factors contributing to IRF4 activation are unknown, IL-1 family members and Th2-related cytokines are potential candidates; it was suggested that IL-1 facilitates IRF4 induction during Th17 differentiation, whereas IL-4 induces IRF4 expression during alternate activation of macrophages (43). Further elucidation of cytokines affecting suppressive capacity of Tregs will help to settle this issue.
Although expression of Bcl6 in Tfh cells is thought to be dependent on Stat 3 activation downstream of IL- 6 and IL-21 (18), it is not clear whether Bcl6 expression in Tregs is dependent on the same cues. Even though loss of Stat 3 in Tregs led to dysregulation of Th17 responses, in that setting, Stat 3 activation was primarily downstream of IL-10R. It is conceivable that in the context of IL-6 and IL-21 signaling, Stat 3 activation in Tregs may lead to Bcl6 upregulation. As Th17 cells can also augment B cell responses (13), a careful analysis is needed to determine whether Stat3-deficient Tregs are impaired in ability to directly control Tfh responses.

\section{Control of sterile inflammation by Tregs}

The concept that inflammation triggers symmetrical activation of factors in effector $\mathrm{T}$ cells and Tregs can be further expanded to different tissue environments; it is easily conceivable that nonimmune tissue- or cell type-specific transcription factors might also be expressed in and have impact on Tregs present in a given tissue, supporting their ability to maintain local homeostasis.

Key evidence in support of this idea came from the identification of a subset of Tregs present in abdominal fat and their role in controlling metabolic inflammation (44). Metabolic disorders such as obesity are often accompanied by low-grade chronic inflammation in the adipose tissue. Recent work demonstrated that Tregs are highly enriched in visceral adipose tissue and their frequency was markedly reduced in several experimental models of obesity, suggesting that antiinflammatory properties of Tregs may feature prominently in control of metabolic syndromes. In contrast to Tregs found in secondary lymphoid organs or other nonlymphoid tissues, fat-resident Tregs were unique in their expression of high levels of an adipocyte lineage transcription factor PPAR $\gamma$ (45). In support of the aforementioned hypothesis, gene expression profiling of these fat-resident Tregs revealed distinct features that mirrored their anatomical location. PPAR $\gamma$ was shown to play an essential role in fat-resident Treg homeostasis and possibly function; PPAR $\gamma$ deficiency in Tregs was associated with a metabolic syndrome resembling type II diabetes, implicating fat-resident Tregs in control of metabolic inflammation. In fact, therapeutic agents such as pioglitazone, which are known to improve several metabolic parameters in obese animals and humans and work as PPAR $\gamma$ agonists, act via modulation of the adipose tissue-resident Treg population (45). Hence, it is plausible that additional tissuespecific transcription factors could also play an important role in regulating Treg function in defined tissue settings.

\section{Stability/plasticity of Tregs}

Since Foxp3 plays a fundamental role in differentiation, homeostasis, and maintenance of suppressor function of Tregs, continued expression of Foxp3 is central to Treg lineage identity. This notion is supported by experiments in which ablation of Foxp 3 in fully differentiated Tregs resulted in a loss of their ability to suppress and in acquisition of immune effector function by "former" Tregs, i.e., production of immune effector cytokines and ability to cause tissue pathology upon transfer into lymphopenic recipients (46). Several reports have indicated that Tregs exhibit marked instability and lose Foxp3 expression under conditions of inflammation and lymphopenia as well as upon vaccination with TLR ligandcontaining adjuvants $(47,48)$. Furthermore, these "ex-Tregs" may convert to pathogenic effector cells and potentially initiate deleterious autoimmune responses. However, these views have been challenged by studies showing that Tregs represent a stable lineage 
and are capable of maintaining identity even under a variety of inflammatory conditions $(49,50)$. Considering ongoing efforts to employ adoptive Treg transfers as a therapy in solid organ transplant patients and in bone marrow transplant patients suffering from graft-versus-host disease (GVHD) (51), the issue of stability of Treg lineage is of considerable practical importance. These disparate findings can be potentially reconciled by envisioning a scenario wherein the observed "plasticity" of Foxp3 expression, instead of reflecting lineage reprogramming in fully differentiated Tregs, is due to the presence of a minor population of recently generated Tregs passing through a transient state of unstable Foxp3 expression. Nevertheless, the ability of inflammatory and tissue environments to alter transcriptional and functional properties of Tregs due to upregulation or activation of transcription factors, which promote effector cytokine responses in conventional $\mathrm{T}$ cells, raises a question as to whether in inflammatory settings some Tregs acquire pathogenic potential regardless of Foxp3 loss. For instance, under conditions of acute toxoplasma infection as well as in cases of skin graft, Tregs gain the ability to produce IFN- $\gamma$ without loss of Foxp3 expression $(52,53)$. Furthermore, the presence of Foxp $3^{+}$ROR $\gamma t^{+}$Tregs that produce IL-17 has been observed in both humans and mice $(54,55)$. Whether cytokines produced by these Tregs have a regulatory role or show an inflammatory function by activating and recruiting other immune cells is currently unclear.

\section{Overriding inhibitions: the molecular view}

As STAT family members play an important functional role in Tregs, their expression and activation state needs to be tightly controlled. While suboptimal Stat expression in Tregs leads to deleterious consequences, aberrant or supraoptimal Stat expression might trigger transcriptional programs that promote

1. Medzhitov R. Inflammation 2010: new adventures of an old flame. Cell. 2010;140(6):771-776.

2. Zhu J, Yamane H, Paul WE. Differentiation of effector CD4 T cell populations $\left({ }^{*}\right)$. Annu Rev Immunol. 2010;28:445-489.

3. Szabo SJ, Sullivan BM, Stemmann C, Satoskar AR, Sleckman BP, Glimcher LH. Distinct effects of T-bet in TH1 lineage commitment and IFN-gamma production in CD4 and CD8 T cells. Science. 2002; 295(5553):338-342.

4. Zhu J, et al. Conditional deletion of Gata3 shows its essential function in $\mathrm{T}(\mathrm{H}) 1-\mathrm{T}(\mathrm{H}) 2$ responses. Nat Immunol. 2004;5(11):1157-1165.

5. Zheng W, Flavell RA. The transcription factor GATA-3 is necessary and sufficient for Th2 cytokine gene expression in CD4 T cells. Cell. 1997; 89(4):587-596.

6. Kurata H, Lee HJ, O’Garra A, Arai N. Ectopic expression of activated Stat 6 induces the expression of Th2-specific cytokines and transcription factors in developing Th1 cells. Immunity. 1999; 11(6):677-688.

7. Veldhoen M, Hocking RJ, Atkins CJ, Locksley RM, Stockinger B. TGFbeta in the context of an inflammatory cytokine milieu supports de novo differentiation of IL-17-producing T cells. Immunity. 2006; 24(2):179-189.

8. Ivanov II, et al. The orphan nuclear receptor RORgammat directs the differentiation program of proinflammatory IL-17+ T helper cells. Cell. 2006; 126(6):1121-1133.

9. Torchinsky MB, Garaude J, Martin AP, Blander JM. Innate immune recognition of infected apoptotic cells directs $\mathrm{T}(\mathrm{H}) 17$ cell differentiation. Nature. 2009; 458(7234):78-82.

10. Ivanov II, et al. Induction of intestinal Th17 cells by segmented filamentous bacteria. Cell. 2009;

inflammatory responses rather than suppression. In this regard, not only loss of Stat1, but also its heightened expression or uncontrolled activation in Tregs due to a loss of miR-146a or SOCS1, respectively, can result in Th1-dependent immunemediated pathology (56). In addition to a sharp increase in IFN- $\gamma$ producing effector T cells, SOCS1- or miR-146a-deficient Tregs also produced IFN- $\gamma$. These results suggest that inflammation sensing in Tregs is tuneable rather than biphasic. It seems likely that excessive activation of STAT family members in Tregs might overcome repression of cytokine genes by Foxp3, leading to acquisition of effector properties, and could negatively affect the set of genes required for suppressor function. These observations suggest a general model of integration of environmental cues by Tregs in which specialized Treg suppression programs are established in dynamically changing inflammatory environments by maintaining an optimal threshold of activation of transcription factors downstream of cytokine receptors crucial for the regulation of corresponding type of immune responses (Figure 2).

\section{Acknowledgments}

We would like to thank members of the Rudensky laboratory for useful suggestions and discussions. This work was supported by grants from the NIH (to A.Y. Rudensky). A. Chaudhry is supported by the Irvington Institute Fellowship Program of the Cancer Research Institute.

Address correspondence to: Ashutosh Chaudhry or Alexander Y. Rudensky, Memorial Sloan-Kettering Cancer Center, 408 E. 69th St., New York, New York 10021, USA. Phone: 646.888.3109; Fax: 646.422.0453; E-mail: chaudha1@mskcc.org (A. Chaudhry); rudenska@mskcc.org (A. Rudensky).

\section{9(3):485-498.}

11. Moseley TA, Haudenschild DR, Rose L, Reddi AH. Interleukin-17 family and IL-17 receptors. Cytokine Growth Factor Rev. 2003;14(2):155-174.

12. Korn T, Bettelli E, Oukka M, Kuchroo VK. IL-17 and Th17 Cells. Annu Rev Immunol. 2009;27:485-517.

13. Hsu HC, et al. Interleukin 17-producing $\mathrm{T}$ helper cells and interleukin 17 orchestrate autoreactive germinal center development in autoimmune BXD2 mice. Nat Immunol. 2008;9(2):166-175.

14. Trifari S, Kaplan CD, Tran EH, Crellin NK, Spits $H$. Identification of a human helper $T$ cell population that has abundant production of interleukin 22 and is distinct from $\mathrm{T}(\mathrm{H})-17, \mathrm{~T}(\mathrm{H}) 1$ and $\mathrm{T}(\mathrm{H}) 2$ cells. Nat Immunol. 2009;10(8):864-871.

15. Duhen T, Geiger R, Jarrossay D, Lanzavecchia A, Sallusto F. Production of interleukin 22 but not interleukin 17 by a subset of human skin-homing memory T cells. Nat Immunol. 2009;10(8):857-863.

16. Ma HL, et al. IL-22 is required for Th17 cell-mediated pathology in a mouse model of psoriasis-like skin inflammation. J Clin Invest. 2008;118(2):597-607.

17. Veldhoen $\mathrm{M}$, et al. The aryl hydrocarbon receptor links TH17-cell-mediated autoimmunity to environmental toxins. Nature. 2008;453(7191):106-109.

18. Crotty S. Follicular helper CD4 T cells (TFH). Annu Rev Immunol. 2011;29:621-663.

19. Fontenot JD, Gavin MA, Rudensky AY. Foxp3 programs the development and function of CD4+CD25+ regulatory T cells. Nat Immunol. 2003; 4(4):330-336.

20. Khattri R, Cox T, Yasayko SA, Ramsdell F. An essential role for Scurfin in CD4+CD25+ T regulatory cells. Nat Immunol. 2003;4(4):337-342.

21. Wildin RS, et al. X-linked neonatal diabetes mellitus, enteropathy and endocrinopathy syndrome is the human equivalent of mouse scurfy. Nat Genet.
2001;27(1):18-20

22. Vignali DA, Collison LW, Workman CJ. How regulatory T cells work. Nat Rev Immunol. 2008; 8(7):523-532.

23. Rubtsov YP, et al. Regulatory $\mathrm{T}$ cell-derived interleukin-10 limits inflammation at environmental interfaces. Immunity. 2008;28(4):546-558.

24. Zheng Y, et al. Regulatory T-cell suppressor program co-opts transcription factor IRF4 to control $\mathrm{T}(\mathrm{H}) 2$ responses. Nature. 2009;458(7236):351-356.

25. Nurieva RI, et al. Transcriptional regulation of th2 differentiation by inducible costimulator. Immunity. 2003;18(6):801-811.

26. Cretney E, et al. The transcription factors Blimp-1 and IRF4 jointly control the differentiation and function of effector regulatory T cells. Nat Immunol. 2011;12(4):304-311.

27. Wang Y, Su MA, Wan YY. An essential role of the transcription factor GATA-3 for the function of regulatory T cells. Immunity. 2011;35(3):337-348.

28. Wohlfert EA, et al. GATA3 controls Foxp $3^{+}$regulatory $\mathrm{T}$ cell fate during inflammation in mice. J Clin Invest. 2011;121(11):4503-4515.

29. Rudra D, et al. Transcription factor Foxp3 and its protein partners form a complex regulatory network. Nat Immunol. 2012;13(10):1010-1019.

30. Chaudhry A, et al. CD4+ regulatory T cells control TH17 responses in a Stat3-dependent manner. Science. 2009;326(5955):986-991.

31. Yang XO, et al. Molecular antagonism and plasticity of regulatory and inflammatory $\mathrm{T}$ cell programs. Immunity. 2008;29(1):44-56.

32. Koch MA, Tucker-Heard G, Perdue NR, Killebrew JR, Urdahl KB, Campbell DJ. The transcription factor T-bet controls regulatory $\mathrm{T}$ cell homeostasis and function during type 1 inflammation. Nat Immunol. 2009;10(6):595-602. 
33. Linterman MA, et al. Foxp $3+$ follicular regulatory $\mathrm{T}$ cells control the germinal center response. Nat Med. 2011;17(8):975-982.

34. Chung Y, et al. Follicular regulatory T cells expressing Foxp 3 and Bcl-6 suppress germinal center reactions. Nat Med. 2011;17(8):983-988.

35. Wollenberg I, et al. Regulation of the germinal center reaction by Foxp $3+$ follicular regulatory $\mathrm{T}$ cells. J Immunol. 2011;187(9):4553-4560.

36. Chang HC, et al. The transcription factor PU.1 is required for the development of IL-9-producing $\mathrm{T}$ cells and allergic inflammation. Nat Immunol. 2010; 11(6):527-534.

37. Staudt $\mathrm{V}$, et al. Interferon-regulatory factor 4 is essential for the developmental program of T helper 9 cells. Immunity. 2010;33(2):192-202.

38. Chaudhry A, et al. Interleukin-10 signaling in regulatory $\mathrm{T}$ cells is required for suppression of Th17 cell-mediated inflammation. Immunity. 2011; 34(4):566-578

39. Saraiva M, O'Garra A. The regulation of IL-10 production by immune cells. Nat Rev Immunol. 2010; 10(3):170-181

40. Koch MA, Thomas KR, Perdue NR, Smigiel KS, Srivastava S, Campbell DJ. T-bet(+) Treg cells undergo abortive Th1 cell differentiation due to impaired expression of IL- 12 receptor $\beta 2$. Immunity. 2012;37(3):501-510.

41. Hall AO, et al. The cytokines interleukin 27 and interferon- $\gamma$ promote distinct Treg cell populations required to limit infection-induced pathology. Immunity. 2012;37(3):511-523.

42. Matsuyama T, et al. Molecular cloning of LSIRF, a lymphoid-specific member of the interferon regulatory factor family that binds the interferonstimulated response element (ISRE). Nucleic Acids Res. 1995;23(12):2127-2136.

43. El Chartouni C, Schwarzfischer L, Rehli M. Interleukin- 4 induced interferon regulatory factor (Irf) 4 participates in the regulation of alternative macrophage priming. Immunobiology. 2010;215(9-10):821-825.

44. Feuerer $M$, et al. Lean, but not obese, fat is enriched for a unique population of regulatory $\mathrm{T}$ cells that affect metabolic parameters. Nat Med. 2009; 15(8):930-939.

45. Cipolletta D, et al. PPAR- $\gamma$ is a major driver of the accumulation and phenotype of adipose tissue Treg cells. Nature. 2012;486(7404):549-553.

46. Williams LM, Rudensky AY. Maintenance of the Foxp3-dependent developmental program in mature regulatory $\mathrm{T}$ cells requires continued expression of Foxp3. Nat Immunol. 2007;8(3):277-284.

47. Zhou X, et al. Instability of the transcription factor Foxp3 leads to the generation of pathogenic memory T cells in vivo. Nat Immunol. 2009;10(9):1000-1007.

48. Sharma MD, et al. Reprogrammed foxp3(+) regulatory $\mathrm{T}$ cells provide essential help to support crosspresentation and CD8(+) $\mathrm{T}$ cell priming in naive mice. Immunity. 2010;33(6):942-954.

49. Rubtsov YP, et al. Stability of the regulatory T cell lineage in vivo. Science. 2010;329(5999):1667-1671.

50. Komatsu N, Mariotti-Ferrandiz ME, Wang Y, Malissen B, Waldmann H, Hori S. Heterogeneity of natural Foxp3+ T cells: a committed regulatory T-cell lineage and an uncommitted minor population retaining plasticity. Proc Natl Acad Sci U S A. 2009; 106(6):1903-1908.

51. Edinger M, Hoffmann P. Regulatory T cells in stem cell transplantation: strategies and first clinical experiences. Curr Opin Immunol. 2011;23(5):679-684.

52. Oldenhove G, et al. Decrease of Foxp3+ Treg cell number and acquisition of effector cell phenotype during lethal infection. Immunity. 2009;31(5):772-786.

53. Sawitzki B, Kingsley CI, Oliveira V, Karim M, Herber M, Wood KJ. IFN-gamma production by alloantigen-reactive regulatory $\mathrm{T}$ cells is important for their regulatory function in vivo. J Exp Med. 2005; 201(12):1925-1935.

54. Zhou L, et al. TGF-beta-induced Foxp3 inhibits $\mathrm{T}(\mathrm{H}) 17$ cell differentiation by antagonizing ROR $\gamma \mathrm{t}$ function. Nature. 2008;453(7192):236-240.

55. Voo KS, et al. Identification of IL-17-producing FOXP3 + regulatory T cells in humans. Proc Natl Acad Sci U S A. 2009;106(12):4793-4798.

56. Lu LF, et al. Function of miR-146a in controlling Treg cell-mediated regulation of Th1 responses. Cell. 2010;142(6):914-929. 